\title{
Pratiques
}

Linguistique, littérature, didactique

175-176 | 2017

Didactiques et médiations des arts et de la littérature

\section{Le prédicat : une question, et une " affaire »}

\section{Alain Rabatel}

\section{OpenEdition}

\section{Journals}

Édition électronique

URL : http://journals.openedition.org/pratiques/3751

DOI : 10.4000/pratiques.3751

ISSN : 2425-2042

\section{Éditeur}

Centre de recherche sur les médiations (CREM)

\section{Référence électronique}

Alain Rabatel, « Le prédicat : une question, et une « affaire » », Pratiques [En ligne], 175-176 | 2017, mis en ligne le 22 décembre 2017, consulté le 05 mai 2019. URL : http://journals.openedition.org/ pratiques/3751; DOI : 10.4000/pratiques.3751

Ce document a été généré automatiquement le 5 mai 2019.

(c) Tous droits réservés 


\title{
Le prédicat: une question, et une « affaire »
}

\author{
Alain Rabatel
}

1 Le vendredi 27 janvier 2017, l'Association des Sciences du Langage (désormais ASL) organisait avant son assemblée générale annuelle une table ronde, comme il est de coutume. Le thème qui avait été choisi à l'automne 2016 portait sur La place des sciences $d u$ langage dans la formation des enseignants (primaire, secondaire, FLE-S): état des lieux et perspectives. Cette table ronde, animée par le président de l'ASL, Alain Rabatel, professeur en sciences du langage à l'Université Claude Bernard Lyon 1 - École supérieure du professorat et de l'éducation (ESPÉ) de l'académie de Lyon -, réunissait Jean-Louis Chiss, professeur en sciences du langage et en didactique du français à l'Université Sorbonne Nouvelle-Paris 3, Jacques David, maître de conférences en sciences du langage à l'Université Cergy-Pontoise et à l'ESPÉ, directeur de la revue Le Français aujourd'hui, Caroline Masseron, professeure émérite en sciences du langage à l'Université de Lorraine, directrice de la rédaction de la revue Pratiques, et Sylvie Plane, professeure émérite en sciences du langage à l'Université Paris-Sorbonne - ESPÉ -, vice-présidente du Conseil supérieur des programmes.

\section{Le prédicat : une, des question(s)}

2 Comme l'indiquait le titre de la table ronde, le sujet était beaucoup plus vaste que la question du prédicat, qui venait de faire irruption dans les médias, brutalement et de façon tonitruante, depuis le début du mois de janvier 2017, comme le rappelle en ouverture de ce dossier S. Plane. Mais, à n'en pas douter, le thème de ces échanges englobait à plus d'un titre cette question vive, en tant que cette nouveauté terminologique touchait :

- aux problématiques des savoirs savants et des savoirs à enseigner, via une réflexion sur la nécessaire transposition didactique des premiers ;

- à la terminologie et à ses évolutions ;

- aux activités à mettre en œuvre pour aborder cette innovation ; 
- au rôle des corps d'inspection dans l'accompagnement des innovations terminologiques et des évolutions des programmes ;

- à la formation initiale et à la formation continuée des enseignants ;

- à la façon dont l'Institution (en l'occurrence, le ministère de l'Éducation nationale - MEN -, mais aussi les rectorats) règle les points précédents, mais également dont elle gère des interrogations professionnelles, des controverses scientifiques, voire des polémiques médiatiques, et donc aux relais qu'elle se donne pour répondre à des inquiétudes légitimes comme aux polémiques de mauvaise foi.

3 Toutes ces questions institutionnelles, scientifiques, professionnelles, forment en définitive une question. Cette situation explique que, alors que l'ASL avait programmé sa table ronde bien avant que n'émerge la polémique, l'association ait ressenti le besoin de rendre public, la veille de la table ronde, le communiqué qu'on lira en annexe, dans lequel elle affirmait souhaiter que le débat public puisse se développer de façon constructive et rationnelle. Et, bien sûr, cela permet de comprendre aussi que la question du prédicat ait pris une place importante dans les débats, même si bien d'autres aspects ont été abordés, comme on le vérifiera en regardant la vidéo de cette manifestation, en ligne : http:// epresence.univ-paris3.fr/7/Watch/3810397.aspx.

Ce média ne peut être affiché ici. Veuillez vous reporter à l'édition en ligne http:// journals.openedition.org/pratiques/3751

Compte tenu des échanges consacrés au prédicat, il est apparu qu'il serait utile d'approfondir la question, sur le plan théorique, comme sur celui des mises en œuvre didactiques en France ou dans la francophonie (Québec, Suisse, Belgique). Il avait donc été convenu, en accord avec la revue Pratiques, de publier fin 2017 un dossier complémentaire sur cette question avec des articles relativement courts, de façon à toucher tous les publics, spécialistes ou enseignants non spécialistes. C'est le résultat de ces travaux qui est publié ici.

6 Qu'il me soit permis de remercier, outre les auteurs, la revue Pratiques qui a joué un si grand rôle pour une didactique du français qui articule savoirs savants (contextualisés) et transposition didactique, avec des activités incarnant les innovations proposées. Évoquer la dimension historique de cet engagement de plus de 40 ans déjà ne nous éloigne guère du prédicat : cela nous rappelle - comme le montrent les contributions de S. Plane et de B. Combettes - combien les innovations ne font sens que si elles sont relayées, éclairées par des activités pédagogiques et didactiques appropriées, faute de quoi les nouveautés les plus pertinentes courent le risque d'être digérées par d'anciennes pratiques...

\section{Le prédicat : une « affaire »}

7 En quel sens peut-on donc dire qu'il y a une " affaire »? D'abord parce que les questions se sont rapidement accumulées dans les médias, dans un contexte bien particulier, celui de l'élection présidentielle, qui a surdéterminé certaines prises de position. La question est devenue une " affaire ", au sens médiatique qu'on donne en général à l'expression. En effet, le prédicat a suscité une couverture importante, tant dans la presse nationale que régionale, dans les radios et télévisions, sur l'internet, à travers un nombre important de prises de position. Fait notable, ces dernières ont émané de professionnels de l'enseignement, de parents, dans des courriers de lecteurs, sur des blogs, des sites 
pédagogiques ou politiques, mais elles ont été aussi le fait de journalistes, qui ont beaucoup écrit sur le sujet, en tentant de faire le point, sollicitant des spécialistes, donnant naissance à un certain nombre de papiers relativement bien informés ${ }^{1}$. Il n'y a donc pas d'«affaire " au sens où il y aurait eu un évènement scandaleux que les médias auraient mal couvert, mais parce qu'une question pédagogique concernant la discipline français a fait l'objet d'une lecture politique, lecture qui n'est pas venue d'abord ni essentiellement de militants politiques - même si ces derniers n'ont pas été inactifs -, mais de professionnels de l'éducation ou d'intellectuels. Ces derniers se sont alarmés de la difficulté d'enseigner une notion nouvelle, inquiétés de l'articulation de cette nouveauté avec des pratiques anciennes (comment enseigner le prédicat et le complément d'objet direct - COD - ou les notions de complément essentiel et de complément de phrase), ou avec la permanence de certaines injonctions (par exemple celle d'enseigner l'accord du participe passé, pour lequel la notion de COD fait sens).

8 Il y a donc une affaire politique ${ }^{2}$, parce qu'il s'est vérifié, une fois de plus, que les innovations dans le domaine de l'enseignement suscitaient des interrogations, des résistances ; que les inquiétudes sont particulièrement vives dès lors qu'elles touchent à l'enseignement du français ; qu'elles se mêlent alors à des craintes sociales ou politiques, pouvant alimenter les discours de la décadence, du nivèlement par le bas... qui s'étaient également manifestés auparavant, autour de la réforme de la formation des maitres et des instituts universitaire de formation des maitres (IUFM)/ESPÉ, des méthodes de lecture, des rythmes scolaires, des évolutions concernant les classes bilangues, notamment, pour ne citer que quelques épisodes des deux derniers quinquennats passés de N. Sarkozy et F. Hollande. On touche là à la dimension politique de l'affaire, avec des discours de l'opposition qui, lorsque la gauche est aux affaires, remettent en cause sa légitimité à réformer dès lors que les innovations sont assimilées à des complaisances envers le pédagogisme et une propension à baisser le niveau pour permettre aux enfants défavorisés une réussite illusoire. Ces discours empruntent parfois aux déplorations des milieux réactionnaires, voire de la fachosphère, et il leur arrive de rencontrer les craintes de professionnels que leur sociologie classerait plutôt à gauche, mais qui, compte tenu de la crise, peuvent être sensibles aux discours qui, sous couvert d'excellence républicaine pour tous, rejoignent finalement les positions conservatrices. Les réactions des professionnels, qui peuvent "mettre beaucoup d'eux-mêmes» dans leurs prises de position, sont comme des symptômes sociologiques et politiques des ratés ou des insuffisances du système de formation - que l'introduction du prédicat était censée partiellement résoudre. Il y a donc « affaire » parce que l'Institution est confrontée à des résistances, des remises en question, à une absence de consensus sur les diagnostics comme sur les remèdes, tant chez les agents de l'Institution que dans la société.

Les indignations des professeurs sur la "grammaire négociable/non négociable » sont le type même de débats tronqués qui ne peuvent cependant pas être rayés d'un trait de plume. S'insurger contre une grammaire négociable, c'est d'abord réaffirmer un désir de scientificité, même si ce dernier repose sur l'idée illusoire que les catégories et notions grammaticales sont indiscutables, établies de toute éternité (voir Raby, 2017). C'est ensuite exprimer la crainte d'une école publique à plusieurs vitesses, avec des maitres exigeants, qui ne négocieraient pas, et d'autres qui abdiqueraient en fonction des difficultés du terrain - qu'on devine liées aux inégalités socioculturelles entre élèves et territoires. C'est enfin renvoyer, à son corps défendant, aux insuffisances de formation des enseignants de français en grammaire, qui focalisent sur les catégories et les 
fonctions parce qu'ils n'ont pas été suffisamment formés pour faire le lien avec la diversité des structures linguistiques et leurs effets sémantiques, en fonction des choix énonciatifs, des stratégies argumentatives et rhétoriques. Ces choix se discutent, ils expliquent ce qui est au cœur des prédications (prédications premières), ce qui relève des prédications secondes (voir Cadiot \& Furukawa, 2000; Roig \& Van Raemdonck, 2014 ; Raby, 2017) : mais ils ne ressortissent pas d'une grammaire négociable au sens où les analyses dépendraient des lubies des enseignants ou du caprice des locuteurs ${ }^{3}$. La langue est une réalité sociale, soumise à des règles, des normes, des conventions (il faut relire Berrendonner, 1982 et Berrendonner, Le Guern \& Puech, 1983). Cependant celles-ci n'opèrent pas toujours en synergie, et donc mettent en difficulté les réponses simples (nature/fonction) mises à mal par la complexité des discours. Car les élèves (comme les maitres) sont confrontés à des énoncés hors normes, à des effets stylistiques en délicatesse avec les règles : il faut expliquer en quoi ils restent (ou non) dans les limites du système, dans quelle mesure ils prennent des libertés avec telle ou telle norme, en fonction des spécificités de la situation, du public, des finalités de leurs prises de parole, et sous quelles conditions ils peuvent être jugés acceptables et «négociés » comme tels. C'est le cas, pour rappeler un exemple bien connu, lorsque des critères entrent en conflit, à l'instar des tensions entre la règle de proximité entre le pronom et son référent et certains choix de progression thématique ${ }^{4}$. C'est pourquoi la formation des enseignants doit leur permettre d'expliquer les règles, les normes et aussi les usages innovants, singuliers ou collectifs, voire de justifier en quoi certaines autres peuvent être dites déviantes, acceptables ou inacceptables. D'où l'importance d'avoir une solide culture grammaticale ${ }^{5}$ qui arme les enseignants comme les élèves d'outils pour comprendre le système, regrouper, hiérarchiser les divers composants des groupes de mots et propositions qui construisent des phrases : le prédicat et la prédication sont de ces outils. Je dis sont, mais sans doute le lecteur pensera qu'il faudrait plutôt dire : étaient. Cette dernière formulation nous fait entrer dans une autre affaire, à laquelle nous étions loin de nous attendre lorsque nous avions lancé l'idée de ce dossier...

\section{« L'affaire dans l'affaire »}

10 L'introduction qu'on vient de lire était en effet bouclée bien avant l'annonce brutale du ministre de l'Éducation nationale d'en finir avec l'enseignement du prédicat, à la miseptembre 2017. C'est, si l'on veut, "l'affaire dans l'affaire », qui confirme le diagnostic que l'affaire était politique, non seulement au sens évoqué ci-dessus, mais par la façon proprement politicienne de donner à croire qu'on règle les problèmes en les niant - comme on croit tuer la fièvre en cassant le thermomètre, en l'occurrence, en abandonnant l'enseignement du prédicat. En effet, la décision de J.-M. Blanquer illustre jusqu'à la caricature l'instrumentalisation de questions éducatives, réduites à des alternatives idéologiques simplistes. Ce serait l'honneur de la politique, en ce domaine comme en d'autres, de ne pas se payer de mots, a fortiori quand les slogans sont vides, ou si peu opposables qu'ils ne veulent pas dire grand chose, sinon qu'ils visent à rendre indignes ceux qui en tiennent pour d'autres conceptions, qui se voient ainsi ravalés à des tenants patentés de l'obscurantisme ${ }^{6}$. Chacun sait qu'une maille à l'endroit et une maille à l'envers font avancer le tricot. Mais la gestion d'une politique éducative a fort peu à voir avec le tricot, et si « défaire, c'est toujours faire », cela n'assure pas toujours d'avancer... 


\section{Présentation des articles du dossier}

11 L'ASL ne se sent en cette affaire porteuse d'aucune vérité officielle, institutionnelle ou scientifique : par ses missions, elle est un lieu d'échanges et de confrontations, comme de défense des enseignants-chercheurs, chercheurs, docteurs et, plus largement, étudiants qui éprouvent de l'intérêt pour les sciences du langage et leur enseignement. C'est pourquoi les lignes qui précèdent se sont bornées à esquisser ce qu'on pourrait appeler un discours de la méthode, pour dégonfler les affaires et traiter des questions vives...

Partant de là, le dossier proposé vise à contextualiser le débat (Sylvie Plane : «Le prédicat est-il subversif ?»), commençant par apporter un témoignage de première main sur la fabrique des programmes, son auteure étant vice-présidente du Conseil supérieur des programmes. Il propose ensuite de comprendre les raisons qui ont présidé aux innovations, aux activités susceptibles de les mettre en œuvre et, en fin de compte, de s'intéresser à des travaux en cours, pour tenter de montrer que bien des craintes exprimées par des enseignants ou des parents n'ont pas lieu d'être. D'où un ensemble d'analyses puisées aux meilleures sources, à la croisée des savoirs savants et de leur transposition didactique. C'est notamment le cas, en France, avec l'article de MarieLaure Elalouf («Utiliser le terme de prédicat: quel cout pour les enseignants? Quels gains pour les élèves?»). La réflexion s'ouvre ensuite à d'autres réalités que l'enseignement de la notion en France. Après tout, n'est-il pas de bonne méthode de sortir $\mathrm{du}$ tropisme franco-français, du discours sur l'exception française (pour le meilleur et parfois pour le pire) et de regarder comment nos collègues de la francophonie essaient de traiter de la question du prédicat (sans forcément en faire toute une affaire) ? D'où trois articles consacrés à l'enseignement du prédicat: au Québec d'abord, avec Carole Fisher et Marie Nadeau («Survivre au prédicat : le cas du Québec »); en Suisse romande, avec un article de Martine Panchout-Dubois, Roxane Gagnon et Ecaterina Bulea Bronckart ("Les péripéties du prédicat en Suisse romande : hier, aujourd'hui et demain...»); en Belgique, enfin, avec la contribution de Dan Van Raemdonck («"Le prédicat m’a tuer”, signé $\mathrm{P}($ articipe) $\mathrm{P}($ assé) »). Pour finir, le dossier élargit la réflexion dans deux directions complémentaires: en premier lieu, sur une mise en perspective théorique et épistémologique de Bernard Combettes qui interroge la notion de prédicat à l'aune de la terminologie et des nécessités de la formation («Le prédicat en question: objectifs d'enseignement, conception de la langue et choix d'une terminologie »), renvoyant ainsi la balle à l'institution, pour qu'elle essaie (encore et toujours) de corriger le tir en y mettant plus de cohérence. En dernier lieu, la livraison de Bernard Colombat invite à un regard épistémologique, revenant sur la question des relations entre le sujet ( hupokeimenon) et «ce qu'on en dit» (kategorêma), aux origines de la réflexion sur le prédicat, à la lumière des relations médiévales entre suppositum - suppôt - vs appositum - ou appôt- («Faut-il réutiliser les concepts linguistiques du passé? Un regard rétrospectif sur le prédicat »).

Tout le dossier qu'on va lire montre qu'il y avait des raisons pour proposer la notion, des raisons aussi de mettre en œuvre des actions ambitieuses, en matière de formation, de communication, pour rectifier des erreurs, mieux faire partager le sens des innovations, fournir davantage d'activités pour aider les enseignants à s'approprier le changement et permettre les articulations avec des savoirs anciens. Comme il est assuré que les 
problèmes restent, nous tentons ci-après de donner des éléments de réflexion qui seront utiles, par-delà les injonctions du temps court.

\section{BIBLIOGRAPHIE}

ARQUEMBOURG, J. (2003). Les temps des événements médiatiques, Paris/Bruxelles, INA/De Boeck. BERRENDONNER, A. (1982). L'éternel grammairien. Étude du discours normatif, Berne, Peter Lang. BERRENDONNER, A., LE GUERN, M. \& PUECH, G. (1983). Principes de grammaire polylectale, Lyon, Presses universitaires de Lyon.

CADIOT, P. \& FURUKAWA, N. (dirs) (2000). « La prédication seconde ». Langue française 127.

RABY, V. (éd.) (2017). « L'énoncé dans les traditions linguistiques : logos, vākya, kalām, oratio et les autres ». Langages 205.

ROIG, A. \& VAN RAEMDONCK, D. (éds) (2014). « Les liaisons de prédications ». Langue française 182.

ROIG, A. \& VAN RAEMDONCK, D. (éds) (2015). « Les liaisons de prédication : lorsque la marque s'estompe ». Langages 200.

\section{ANNEXES}

\section{Communiqué de l'ASL à propos de la polémique sur le prédicat}

L'Association des Sciences du Langage observe non sans inquiétude que l'École fait l'objet d'une nouvelle polémique sur les contenus d'enseignement à propos de ce que d'aucuns nomment « l'affaire du prédicat ». Cette situation appelle plusieurs commentaires sur le fond comme sur la forme.

\section{Sur le fond}

Le prédicat n'est pas une idée farfelue qui aurait germé dans le cerveau-enflammé-depédagogues-ou-de-didacticiens-acharnés-à-compliquer-la-vie-des-élèves-et-de-leursparents : le site de publications scientifiques Persée recense pas moins de 6577 articles sur le prédicat!

Certes, la notion est complexe : ce n'est pas un fait observable comme le sont des traits qui servent à distinguer objectivement tel son de tel autre, son objectivation dépend du cadre théorique qui le pense. Alors que le prédicat logique (remontant à l'Antiquité) distinguait ce qu'on dit du sujet de ce dont on parle (sans faire la distinction entre des compléments essentiels et d'autres non essentiels), le prédicat grammatical reprend à son 
compte la séparation entre ce dont on parle et ce qu'on en dit, tout en séparant la fonction du groupe verbal du groupe complément de phrase. Le prédicat grammatical se construit autour du verbe et est constitué de tous les éléments sous sa rection, indépendamment des éléments qui appartiennent au groupe sujet et au groupe complément de phrase (syntaxiquement déplaçable, supprimable, etc. malgré l'importance des informations sémantiques apportées).

Cette notion est utile pour la compréhension par les élèves du fonctionnement grammatical de leur langue. En effet, le verbe désigne une classe de mots (comme celle des adjectifs, des noms...) tandis que le prédicat désigne une fonction syntaxique. Il offre un cadre intégrateur permettant de le distinguer des autres grands groupes fonctionnels de la phrase puis, dans une phase ultérieure, de procéder à des analyses plus détaillées de leurs composants. C'est pourquoi le Canada a depuis plusieurs années introduit ce « nouveau » métalangage sans que cela n'ait produit les effets catastrophiques annoncés par d'aucuns.

\section{Sur la forme (du débat public)}

On ne saurait tenir pour rien le travail des spécialistes, linguistes et didacticiens, au fait des recherches portant sur l'enseignement de la langue, qui ont participé à l'élaboration des programmes, qui ont été très attentifs aux questions de terminologie grammaticale, à des fins scolaires. Les projets de programmes ont été soumis aux organisations professionnelles, puis à une consultation nationale, enfin au Conseil Supérieur des Programmes auquel participent les organisations syndicales, avant d'entrer en vigueur à la rentrée 2016.

Assurément, les innovations terminologiques sont délicates. Elles nécessitent des documents d'accompagnement (qui existent), une formation initiale et continue des enseignants qui sont, par nature, toujours perfectibles. Il y a sans doute à progresser ici, mais le travail est en cours, et il est en ces matières important de se donner du temps (et des moyens) pour que les innovations soient bien comprises dans leurs enjeux.

Cependant, on voit se répandre dans les médias comme sur internet des propos émanant de personnes peu informées ou de mauvaise foi, menant une campagne malhonnête sur le thème qu'on n'enseignerait plus la grammaire, que tout serait « négociable ». Alors que les rumeurs, les amalgames, les mensonges ont joué un rôle détestable dans l'élection présidentielle américaine, il ne faudrait pas que ces polémiques malhonnêtes viennent abaisser le débat légitime qu'appelle, notamment sur les questions éducatives, la future élection présidentielle française.

Paris, le 26 janvier 2017

\section{NOTES}

1. Le lecteur trouvera dans l'article de S. Plane l'essentiel des liens suscités par l'affaire et, dans celui de M.-L. Elalouf, un certain nombre de réponses à des préoccupations exprimées dans des textes d'enseignants dont les liens figurent en note. 
2. C'est cette dimension qui fait utiliser la notion d'affaire, plutôt que celle, fréquente en analyse de discours et dans les sciences de l'information et de la communication, d'évènement médiatique (Arquembourg, 2003), même si l'affaire est aussi un évènement médiatique.

3. On serait mieux inspiré de rappeler combien les manipulations (tests) de formes (un $\mathrm{N}$ ou le $\mathrm{N}$, par exemple, dans un contexte minimum) font appel à des «jugements» (des intuitions) de sens ou de grammaticalité qu'il convient de confronter, d'étayer, et, finalement, de " graduer ». S'il y a «négociation » c'est que les formes langagières sont soumises à variation et que les locuteurs sont porteurs d'une "grammaire intuitive» elle-même variable et dont les justifications méritent explicitation collective.

4. Ainsi, le pronom il, dans la phrase suivante, «Le ballon a traversé la route, renversé le seau puis il a roulé encore quelques mètres ", n'est pas mal construit, en dépit de la règle selon laquelle le pronom ne doit pas être éloigné de son antécédent, surtout lorsqu'il y a un risque de confusion avec un mot de même genre que le référent (c'est le cas de seau par rapport à ballon). En l'occurrence, il n'y a pas de confusion possible, en raison du sémantisme (un seau roule difficilement, encore que ce ne soit pas impossible) mais surtout à cause de la continuité des rôles syntaxiques et de la progression thématique à thème constant, «le ballon " étant systématiquement en position sujet, et en tête de proposition. Aussi tout le monde comprend que il ne renvoie qu'à ballon, et pas à seau et tout le monde trouvera cette phrase bien formée, compte tenu des principes d'organisation qui la régissent et de l'intention communicative qui émerge de l'énoncé, mettant en avant une suite d'actions effectuées par le même actant.

5. C'est loin d'être suffisamment le cas, et l'institution ne peut pas s'exonérer de ses responsabilités.

6. "Je suis dans un travail d'organisation de l'Éducation nationale pour que tous les enfants sachent lire, écrire, compter et respecter autrui à la sortie de l'école primaire. Si cela gêne M. Lussault [le directeur démissionnaire du Conseil supérieur des programmes, opposé à l'abandon du prédicat, voir infra l'article de S. Plane], ce n'est vraiment pas grave qu'il s'en aille », (J.-M. Blanquer sur RTL, 25/09/2017).

\section{AUTEUR}

\section{ALAIN RABATEL}

Université Lyon 2, Centre national de la recherche scientifique, École normale supérieure de Lyon, Icar, UMR 5191, F-69342, France 